\title{
BMJ Open Use of a patient-centred educational exchange (PCEE) to improve patient's self-management of medicines after a stroke: a randomised controlled trial study protocol
}

Judith Ann Coombes, ${ }^{1,2}$ Debra Rowett, ${ }^{3,4}$ Jennifer A Whitty, ${ }^{1,5}$ W Neil Cottrell ${ }^{1}$

To cite: Coombes JA, Rowett D, Whitty JA, et al. Use of a patient-centred educational exchange (PCEE) to improve patient's self-management of medicines after a stroke: a randomised controlled trial study protocol. BMJ Open 2018;8:e022225. doi:10.1136/ bmjopen-2018-022225

- Prepublication history and additional material for this paper are available online. To view these files, please visit the journal online (http://dx.doi org/10.1136/bmjopen-2018022225).

Received 25 April 2018 Accepted 13 July 2018

Check for updates

(c) Author(s) (or their employer(s)) 2018. Re-use permitted under CC BY-NC. No commercial re-use. See rights and permissions. Published by BMJ.

${ }^{1}$ School of Pharmacy, University of Queensland, Brisbane,

Queensland, Australia

${ }^{2}$ Pharmacy Department,

Princess Alexandra Hospital,

Brisbane, Queensland, Australia

${ }^{3}$ School of Pharmacy and

Medical Sciences, University of

South Australia, Adelaide, South

Australia, Australia

${ }^{4}$ DATIS, Southern Adelaide Local Health Network, Flinders Medical Centre, Adelaide, South Australia, Australia

${ }^{5}$ Norwich Medical School, University of East Anglia, Norwich, UK

Correspondence to Judith Ann Coombes; judith@pharmacy.uq.edu.au

\section{ABSTRACT}

Introduction National and international guidelines make recommendations for secondary prevention of stroke including the use of medications. A strategy which engages patients in a conversation to personalise evidence-based educational material (patient-centred educational exchange; PCEE) may empower patients to better manage their medications.

Methods and analysis This protocol outlines a non-blinded randomised controlled trial. Consenting patients admitted with a diagnosis of stroke or transient ischaemic attack will be randomised 1:1 to receive either a PCEE composed of two sessions, one at the bedside before discharge and one by telephone at least 10 days after discharge from hospital in addition to usual care (intervention) or usual care alone (control). The primary aim of this study is to determine whether a PCEE improves adherence to antithrombotic, antihypertensive and lipid-lowering medications prescribed for secondary prevention of stroke over the 3 months after discharge, measured using prescription-refill data. Secondary aims include investigation of the impact of the PCEE on adherence over 12 months using prescriptionrefill data, self-reported medication taking behaviour, selfreported clinical outcomes (blood pressure, cholesterol, adverse medication events and readmission), quality of life, the cost utility of the intervention and changes in beliefs towards medicines and illness.

Ethics and dissemination Communication of the trial results will provide evidence to aid clinicians in conversations with patients about medication taking behaviour related to stroke prevention. The targeted audiences will be health practitioners and consumers interested in medication taking behaviour in chronic diseases and in particular those interested in secondary prevention of stroke. The trial has ethics approval from Metro South Human Research Ethics Committee (HREC/15/ QPAH/531) and The University of Queensland Institutional Human Research Ethics (2015001612).

Trial registration number ACTRN12615000888561; Preresults.

\section{INTRODUCTION}

Stroke is one of the leading causes of death worldwide. $^{12}$ About a third of those who
Strengths and limitations of this study

- The design of randomising participants to the patient-centred educational exchange will provide the opportunity to take into account other changes, which may occur across the time of the study.

- The use of questionnaires, validated as research tools, to elicit patient perceptions will be integrated with the approach used in 'academic detailing'.

- The strength of the intervention is that it is underpinned by a combination of theories of behavioural change.

- This study links the use of both prescription refill data as an objective adherence measurement and patient self-reported adherence.

- As is common with many behavioural intervention studies, this study is not blinded once the participant has been allocated to either the intervention or control group, which may introduce bias to the study.

suffer from a stroke die within 28 days and a further third are left permanently disabled placing a burden on themselves, their family and the community. ${ }^{34}$ After an initial stroke the cumulative incidence of a subsequent stroke is about $30 \%$, with the highest occurrence in the first 12 months $(12 \%) .^{5-7}$ In an individual experiencing a transient ischaemic attack (TIA) or a minor stroke $(<3$ on the National Institutes of Health Stroke Scale ${ }^{8}$ ), the 30-day incidence of stroke is $11 \%-15 \% .^{9}$ After either a stroke or TIA, Internationa $1^{10-12}$ and Australian guidelines ${ }^{13}$ recommend secondary prevention strategies. Recommendations include the use of antithrombotic therapy, medications for blood pressure (BP) lowering and cholesterol-lowering medications. The high rate of recurrence in the first weeks and months of a minor stroke or TIA emphasises the importance of early initiation and subsequent persistence to secondary prevention medicines to reduce 
the risk of subsequent stroke. ${ }^{9}$ Stroke survivors may not benefit due to poor adherence to the medications ${ }^{14-16}$ or the benefit may be offset by the occurrence of adverse drug events. ${ }^{17}$ Reports of patient adherence to secondary prevention medications vary widely ranging from $40 \%^{14}$ to $86 \%{ }^{15}$ and are influenced by the timing and method of measurement. There are many reasons reported for reduction in adherence including: lower income, multiple comorbidities, minor stroke or TIA,${ }^{18}$ forgetfulness, trivialising stroke and low necessity beliefs in taking medications. ${ }^{19}$

Educational interventions focused on improving patient use of medications for secondary prevention of stroke have shown impact on patients' knowledge, but other outcome measures have had varied results. ${ }^{20-22}$ Debate centres on whether a change in knowledge will result in a change of medicine-taking behaviour or whether alternative approaches such as addressing necessities and concerns about medication, ${ }^{23}$ agreeing goals or providing key messages about medication taking will be more effective in changing behaviour. Previously validated questionnaires have been used to identify patients' perceptions of their illness, ${ }^{24}$ beliefs about medications ${ }^{25}$ and medication-taking behaviour, ${ }^{26}$ and these have been used to provide a structure to encourage patient input into a personalised intervention. ${ }^{27}$ Another approach to empower patients in medication-related self-management has incorporated 'academic detailing' ${ }^{28} 29$ also described as 'educational visiting, ${ }^{30}{ }^{31}$ Academic detailing uses a social marketing framework, to encourage information exchange while delivering key messages in order to influence behaviour. The approach includes the following key features: identifying baseline knowledge and motivations for medication use, defining clear educational and behavioural objectives, establishing credibility, referring to authoritative sources of information, and presenting both sides of controversial issues, stimulating participation in educational interactions, using concise graphical educational materials, highlighting and repeating the essential messages and providing positive reinforcement of improved practices in follow-up communication. ${ }^{28}$

Combining these two strategies, identifying patients perceptions' and beliefs' then using these to personalise educational messages and to engage patients in a conversation, may empower patients to better manage their medications. This approach will be referred to as a patient-centred educational exchange (PCEE). The PCEE has been tested for feasibility, and was found to be acceptable to the participants, manageable for the healthcare professional and the beliefs and perceptions elicited by the questionnaires were able to be used to personalise the conversation..$^{32} \mathrm{~A}$ limitation of this feasibility study was that because the researcher delivered the intervention, the training requirements, use of resources and opinions of staff were not evaluated. The impact of the PCEE on patient self-management of stroke prevention medications has yet to be determined.

\section{Aim}

The primary aim of this study is to determine whether a PCEE improves adherence to antithrombotic, antihypertensive and lipid-lowering medications prescribed for secondary prevention of stroke over the 3 months after discharge, measured using prescription refill data.

Secondary aims include: investigation of the impact of the PCEE on adherence over 12 months using prescription refill data, self-reported medication-taking behaviour, self-reported clinical outcomes (BP, cholesterol, adverse medication events and readmission), quality of life, the cost utility of the intervention and changes in beliefs towards medicines and illness.

To address these aims, we will conduct a randomised controlled trial (RCT), with an intervention comprised of two PCEE sessions: one before discharge from hospital and one by telephone at least 10 days after discharge.

\section{METHODS AND ANALYSIS}

This protocol was developed in accordance with the Standard Protocol Items: recommendations for intervention trials (SPIRIT) statement (see online supplementary file 1. SPIRIT checklist).

\section{Study design}

This study is a non-blinded RCT. Participants will be randomised 1:1 to either the intervention group (intervention and usual care) or the control group (usual care).

\section{Setting}

The setting will be the 'Medical Stroke Unit' (MSU) or the Medical Assessment and Planning Unit (MAPU), of an Australian tertiary referral hospital.

\section{Study population}

\section{Inclusion criteria}

Participants recruited to this study must be aged 18 years or older, have been admitted to the MSU or the MAPU with a principal diagnosis of stroke or TIA, and are planned to be discharged to their home.

The participant should be expecting to manage their own medication after discharge home, have a documented Mental Status Questionnaire (MSQ) ${ }^{33}$ score of $10 / 10$ at the time of recruitment and be able to provide consent. The consent form requires the researcher to sign a declaration saying that they have given a verbal explanation of the research project, its procedures and risks, and believe that the participant has understood that explanation. This means the participant is unlikely to have severe problems with verbal communication.

\section{Exclusion criteria}

Those patients planned for discharge to a residential care facility (eg, a nursing or residential care home) where a staff member is responsible for the patients' medication administration, those patients who have been planned for a rehabilitation period of greater than 1 month as they will be having weekly education sessions, those with an 
MSQ $<10$, unable to complete the questionnaire (even) with assistance (this may be due to language difficulties or cognitive impairment) and those who do not provide consent. Those who are excluded will receive standard care, which includes education, without incurring any disadvantage.

\section{Patient and public involvement}

A feasibility study ${ }^{32}$ was conducted to inform the design of the PCEE used in this RCT. Of the 18 participants, 10 completed an evaluation of the proposed intervention which resulted in changes to the final protocol. This included improved graphics to be used in this study, the use of mobile telephones with messaging to facilitate follow-up calls, and bridging sentences between questionnaires and the use of the infographic. With respect to the burden of the intervention, 7 of the 10 indicated that the session was not too long or too short and 9 of 10 agreed that the materials helped them. The participants were invited to ask a questions and prompted to discuss previous experiences as part of the feasibility study, this has been included in the current protocol.

Patients and public were not involved in development of the research question or outcome measures, they will not be involved in the recruitment or ongoing conduct of the current study. The participants will be given contact details to request the results of the study.

\section{Recruitment}

All admissions to MSU and MAPU will be screened using 'bed lists' for 5 days of every week. Those admissions with a diagnosis of stroke or TIA will be further screened for a documented MSQ of 10/10 and plan for further rehabilitation or discharge to home. The researcher will then approach the potential participants on the ward to determine whether they are willing to participate in the study.

\section{Allocation/randomisation}

The allocation of participants to control or intervention will be concealed until the participant has been consented to reduce allocation bias. After the participant has consented to the study, the research pharmacist will contact the clinical trials pharmacist, who is not involved in the study and who will identify the allocation, one to one, to either the intervention or control group. The allocation will be previously determined using a computer-generated four block randomisation code using Sealed Envelope. ${ }^{34}$ The allocation will be concealed by placing the allocation in sealed opaque envelopes stored in the clinical trials office of the pharmacy department.

Once the participant is allocated, the researcher will no longer be blinded to participant's allocation. The reason the researcher will no longer be blinded is that the researcher will conduct the intervention and follow-up calls.

\section{Sample size}

The primary outcome is adherence measured by the proportion of days covered (PDC) (defined as the days of medication supplies when the medications were collected divided by the days in the time interval) over the 3 months after discharge, using prescription refill data for three classes of medications (antithrombotic, antihypertensive and lipid-lowering medications).

The sample size calculation used the criterion for significance (alpha) set at 0.05 and the power (beta) at $80 \%$. It is proposed that the intervention will result in a $7 \%$ improvement in adherence compared with standard care. This difference of $7 \%$ was selected as reasonable; because an effect of this magnitude has been shown with secondary prevention medications used for cardiovascular diseases ${ }^{35} 36$ and has been linked to a clinical difference. ${ }^{37}$ An effect size of $0.54(0.07 / 0.13)$ was selected using results from a study conducted with participants discharged on similar medications after a diagnosis of acute coronary syndrome. ${ }^{36}$ A sample size of 55 in each arm is required for effect size of 0.54 . We allowed for a slighter larger pooled SD of 0.15 (effect size $0.7 / 0.15=0.47$ ) requiring a sample size of $n=73$. Adherence data are likely to be skewed ${ }^{38}$ and so will not fulfil the requirements for a parametric test. Lehmann and $\mathrm{D}^{\prime}$ Abrera ${ }^{39}$ suggest the addition of $15 \%$ more participants $(\mathrm{n}=84)$ when planning to use non-parametric tests such as the Mann-Whitney U test. Assuming attrition rates of approximately $10 \%$, we would need to enrol at least 92 participants for each group; we propose to include 100 participants in each arm. It is predicted that approximately two participants will be recruited per week, estimating a 2-year recruitment period. The first participant was recruited on the 18 December 2015 and the study will be ongoing until April 2019.

\section{Procedure}

Both the intervention and control group will receive usual care. In addition to usual care participants in the intervention group will receive two sessions of a 'PCEE', one before discharge and one by telephone at least 10 days after discharge. These sessions will be conducted by a clinical pharmacist who attends weekly multidisciplinary MSU meetings, has a postgraduate qualification in clinical pharmacy (MSc ClinPharm) and training in academic detailing. In this study, the intervention pharmacist will also be collecting the study data.

\section{Usual care}

Usual care includes admission to a stroke-specific ward, multidisciplinary care by the stroke team, education using Stroke Foundation-Australia materials by the stroke nurse, ${ }^{40}$ clinical pharmacy services provided by the ward pharmacist and discharge advice provided by the medical staff. Usual care provided by the ward pharmacist includes medication history taking and reconciliation, medication review during the admission, discharge reconciliation, provision of a medication list ${ }^{41}$ and medication counselling at discharge. 


\section{Control group}

The control group will receive usual care as described above.

\section{Intervention: PCEE}

The intervention consists of two sessions, one which will take place at the patients' bedside before the usual pharmacist discharge counselling and the second which will be conducted over the telephone at least 10 days after discharge. These sessions are additional to and designed to integrate with usual care. The PCEE is structured with an introduction, conversation and conclusion.

The session begins with an 'introduction' phase establishing credibility 'I am a pharmacist with an interest in patients taking medication to reduce the risk of stroke.' Next, the clinical pharmacist will give the opportunity to the patient to ask a question. 'What one thing would you most like to discuss about medications you have been prescribed since your stroke/TIA?' There is an opportunity to answer this question before moving on.

The session will then move into the 'conversation' using previously validated questionnaires to identify patients' perceptions, beliefs and concerns about their stroke in general (using the Brief-Illness Perception Questionnaire (Brief-IPQ) ${ }^{24}$ and medications in particular (using the Beliefs about Medicine Questionnaire specific (BMQ-specific). ${ }^{25}$ There is also an opportunity for the patient to self-report their previous medication taking behaviour for the medications of interest (using the Medication Adherence Questionnaire (MAQ) ${ }^{26}$ The identified barriers and enablers will be used to personalise the conversation. A double-sided single page document will be personalised and given to the participant (the detailing tool). The detailing tool contains an infographic to help illustrate the discussion about the stroke prevention medications the patient has been prescribed on one side, and four a priori key messages on the other side (see online supplementary file 2. infographic example).

The four key messages are: 'Know about your medications prescribed to reduce risk of stroke', 'Organise ongoing supply of your medications', 'Continue to take these medications as agreed with your doctors' and 'Report any new symptoms or concerns to your doctor'.

In the final phase, 'conclusion', items identified to be discussed when the clinical pharmacist telephones the patient will be listed.

To provide an opportunity for 'follow-up' and reinforcement of key messages, the intervention is designed to include two sessions. The clinical pharmacist will arrange to telephone the participant at least 10 days after discharge to ask them the same questions and to talk about their medications.

It is hypothesised that patients in the intervention group will be influenced to organise ongoing supply of their medications and take their medications as prescribed. In addition, it is hoped that if they identify that they are experiencing unwanted effects from a medication(s), they will not keep taking medication(s) long term, rather discuss their concerns with their doctor.

\section{Outcomes}

The primary outcome is adherence measured by the PDC over the 3 months after discharge, using prescription refill data (obtained from the Pharmaceutical Benefits Scheme; PBS) for the combination of up to three classes of medications (antithrombotic, antihypertensive and lipid-lowering medications) prescribed.

Secondary outcomes include:

- Adherence measured by the PDC over the 12 months after discharge, using prescription refill data for up to three classes of medications (antithrombotic, antihypertensive and lipid-lowering medications).

- Self-reported medication adherence (measured using the MAQ), organising of ongoing medication supply, and medication-taking behaviour and communication to prescriber in response to perceived medication-related adverse events.

- Self-reported changes between baseline and 3 and 12 months in perception to their illness (stroke) using Brief-IPQ and changes between baseline and 3 and 12 months in beliefs about medications for stroke prevention using BMQ-specific.

- Clinical outcomes:

- Medication-related adverse events (identified by self-report or hospital readmission).

- Self-report of BP results.

- Self-report of cholesterol level.

- Readmission to hospital with stroke and/or myocardial infarction (MI) (identified by self-report or hospital records).

- Changes from baseline to 3 and 12 months in self-reported quality of life using the EuroQol 5 dimension, 5 level quality of life tool (EQ-5D-5L). ${ }^{42} 43$

- Cost-utility analysis using a ratio of incremental cost (cost of the PCEE intervention compared with usual care) to incremental benefit (change in quality-adjusted life years (QALYs). ${ }^{44}$

The complex relationship between the intervention described here and the measures of the impact we expect to make has been described by authors of previous studies. ${ }^{4546}$ Table 1 categorises the study outcome measures by outcome type and who measures it.

\section{Behavioural measures}

Behavioural measures look at things the participant has done. In this study visiting the doctor and having their medication dispensed is observer collected. ${ }^{47} 48$ Pharmaceutical claims data can provide an objective, non-invasive measure of adherence and has been used in many drug trials and in a number of studies similar to this one. A range of methods for use of claims data to measure medication possession ratios and PDC have been described to assess an individuals' medication adherence. ${ }^{38} 47-50$

The PDC is defined as the days of medication supplies when the medications were collected divided by the days 
Table 1 The proposed measures categorised by outcome type and who measures it

\begin{tabular}{|c|c|c|c|c|}
\hline & Behavioural outcomes & $\begin{array}{l}\text { Affective-cognitive outcomes: (this } \\
\text { includes knowledge, understanding, } \\
\text { satisfaction) }\end{array}$ & Health outcomes & Economic outcomes \\
\hline \multirow[t]{3}{*}{$\begin{array}{l}\text { Patient } \\
\text { self- } \\
\text { reported }\end{array}$} & $\begin{array}{l}\text { Self report of adherence } \\
\text { using MAQ }\end{array}$ & $\begin{array}{l}\text { Participant ability to identify each } \\
\text { medication of interest along, reason for } \\
\text { use when answering the MAQ }\end{array}$ & $\begin{array}{l}\text { Blood pressure, } \\
\text { cholesterol levels }\end{array}$ & \\
\hline & $\begin{array}{l}\text { Self-report of organising } \\
\text { medication supply }\end{array}$ & $\begin{array}{l}\text { Participant knowing their: blood pressure, } \\
\text { cholesterol level }\end{array}$ & $\begin{array}{l}\text { Blood pressure, } \\
\text { cholesterol levels }\end{array}$ & \\
\hline & & & Self reported ADE & \\
\hline \multirow[t]{2}{*}{$\begin{array}{l}\text { Observer } \\
\text { collected }\end{array}$} & $\begin{array}{l}\text { Visit to doctor-Medicare } \\
\text { data }\end{array}$ & & $\begin{array}{l}\text { Readmission/admission } \\
\text { for ADE }\end{array}$ & $\begin{array}{l}\text { Readmission/admission } \\
\text { for } A D E(s)\end{array}$ \\
\hline & $\begin{array}{l}\text { Prescription refills- } \\
\text { pharmaceutical benefits } \\
\text { scheme data }\end{array}$ & & Events-stroke, MI & Events-stroke, MI \\
\hline
\end{tabular}

Adapted from Shay and Lafata. ${ }^{45}$

ADE, adverse drug event; BMQ specific, Beliefs about Medicine Questionnaire specific ${ }^{25}$; Brief-IPQ, brief IIIness Perception Questionnaire ${ }^{24}$; EQ-5D-5L, Quality of Life Measure ${ }^{42}$; MAQ, Medication Adherence Questionnaire ${ }^{26}$; MI, myocardial infarction.

in the time interval. This can be averaged over the total (eg, a 90-day interval), or to be more reflective of medication exposure, a shorter interval can be measured and added together (eg, in Australia most medications for chronic diseases are supplied at approximately 1-month intervals). The PDC calculated using multiple short intervals $^{49}{ }^{50}$ can more accurately account for gaps in supply or extra medication supplies. The example shown in figure 1 adapted from Bijlsma $e t a l^{49}$ and Bryson et $a \tilde{l}^{0}$ shows how the adherence over three lots of 30-day intervals can be calculated using the gaps in supply for three patients obtaining 30-day supplies. The calculation used is: Proportion of 90 days covered $=(90-$ total of days not covered in each 30 -day interval) $/ 90 \times 100$.

Patient 1 obtained medications on the day of discharge, 30 days later and then had a gap of 5 days before the third

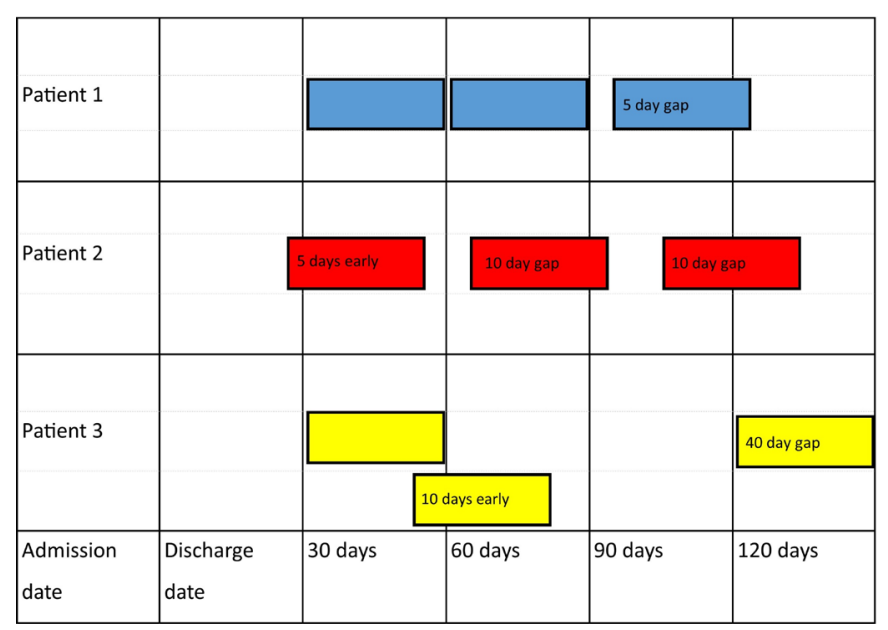

Figure 1 Examples of proportion of days covered calculated using multiple 30-day intervals. supply. Patient $1 \mathrm{PDC}=[(30+0)+(30+0)+(30-5)] / 90 \times 100$ $=94 \%$.

Patient 2 obtained the first supply 5 days before discharge, these were not used until the day of discharge so although the second supply was obtained 10 days after the first supply, there was only a 5-day gap in supply for the patient. Five days of tablets remaining from the second supply were used in the third interval. The third supply was obtained after a 10-day gap. Patient 2 $\mathrm{PDC}=[(30+5-5)+(30-5)+(30+5-10)] / 90 \times 100=89 \%$.

Patient 3 obtained medications on the day of discharge, 20 days later and then had a gap of 40 days before the third supply. Patient 3 PDC= $[(30+0)+(30+10-10)+(30-30)] / 90 \times 100=67 \%$.

In this study the days covered in each dispensing interval will be calculated for up to three different medications (antithrombotic, antihypertensive, lipid-lowering medication) dependant on the medication plan at discharge. These will then be expressed as a mean (across the up to three medications) percentage and analysed as a continuous variable.

Self-reported behavioural measures in this study include self-report of medication adherence using the MAQ ${ }^{26}$ organising an ongoing medication supply and action taken if they experience adverse effects from their medication. The MAQ is a well-validated scale, previously used in many clinical conditions. ${ }^{51}$

\section{Affective cognitive outcomes}

Affective cognitive outcomes include measures of what the participant knows and how they feel, these are usually self-reported.

In this study, the affective cognitive group includes metrics such as knowledge and understanding regarding 
name, type and dose of medications, participants knowing their BP reading or cholesterol level, participants' perceptions of their illness and beliefs about their medications. Participant perceptions of their stroke will be evaluated using the adapted-Brief IPQ at baseline, 3 and 12 months. Beliefs about antithrombotic, antihypertensive and lipid-lowering medications will be evaluated using the BMQ-specific at baseline, 3 months and 12 months. Changes in these may be able to be used to explain changes in other measures for example adherence. ${ }^{46}$

\section{Health outcomes}

In this study, we will evaluate patient self-reported clinical measures (BP and Cholesterol), readmissions, subsequent stroke or MI, adverse drug reactions and quality of life.

Medication-related adverse events will be identified by patient self-report using probe questions adapted from a previously validated trigger tool ${ }^{52}$ at 3 months and 12 months for each medication class of interest. Medication-related adverse events will also be collected from any readmission notes at 3 months and 12 months postdischarge.

Quality of life will be measured using EQ-5D-5L ${ }^{42}$ before discharge (baseline), 3 months and 12 months. This tool (EQ-5D-5L) has previously been used in stroke research. $^{53}$

\section{Economic outcomes}

Economic outcomes will be determined from the health service perspective using an incremental cost-effectiveness ratio (ICER). The ICER indicates the difference between the intervention and control cost (time and resources costed) per the difference in QALYs determined using the quality of life measure EQ-5D-5L. Time taken to deliver the PCEE sessions (intervention group only) will be recorded on the interview schedule at both the bedside (before discharge) and telephone follow-up (7-10 days post discharge) sessions and costed using standard pharmacist salary rates. Any impact of the intervention on health resource use (eg, medication use, hospital readmissions) will be considered when estimating costs. Patient interview using EQ-5D-5L will be conducted before discharge (baseline), at 3 months and 12 months. Uncertainty in the estimated ICER will be evaluated using non-parametric bootstrapping techniques.

\section{Data collection}

A schedule of assessments including the timing for data collection is shown in table 2. Data will be collected by the investigator prior to the patient's discharge, at least 10 days after discharge (intervention group only), at 3 months and at 12 months.

Demographic data collected prior to the patients discharge include patient age, sex, stroke type, whether they have had a previous stroke, whether they live alone, cholesterol levels and BP on discharge. The demographic data are required to describe the population in the study and to ensure the intervention and usual care groups are comparable.

Participant self-reported data will be obtained by the researcher conducting telephone follow-up using the telephone numbers they provide during consent. If the participant does not answer the first call and has provided a mobile telephone number, the researcher will send a text message using the study mobile phone asking for a 'good time to talk'. The protocol allows for a total of three attempts to contact the participant for follow-up calls.

\section{Data management}

Data will be entered electronically from the case record forms using a study number with no identifying information into Microsoft Excel and SPSS Statistics V.25 both stored on a password-protected computer. In all reports from this research, information will be provided in such a way that the participant cannot be identified. Data entry and analyses will be performed using Microsoft Excel and SPSS Statistics V.25.

\section{Data analysis}

An intention-to-treat analysis will be conducted. Results will be reported as numbers and percentages for categorical variables and means (SD) or medians (IQR) for continuous variables. Demographic data and baseline characteristics in the intervention and control groups will be compared using descriptive statistics. Outcomes and changes in outcomes (from baseline) will be compared at 3 months and 12 months.

Adherence measured using the PDC from the prescription refill data will be compared using the Mann-Whitney $\mathrm{U}$ two-sided test. Changes from baseline in quality of life, perceptions of illness and beliefs about medicines will be analysed using the Mann-Whitney $\mathrm{U}$ test. Adherence by self-report, medication-related adverse events and readmissions will be analysed using the $\chi^{2}$ test.

\section{Adverse event reporting and quality assurance}

This study involves completing a questionnaire and discussing stroke medications through one face-to-face interview and three follow-up telephone calls for the intervention group. The control group will complete one face-to-face interview and two follow-up telephone calls. It is possible that during either the face-to-face interview or one of the telephone interviews, the participant identifies a medication-related issue. Although this is unlikely to be as a result of the study, the researcher may still have concerns over the patients' safety. If the researcher has concerns requiring immediate intervention, the patients' doctor will be contacted. In the case of the face-to-face interview in the hospital that will be a medical member of the treating team. In the case of the telephone interview that will be the patients' general practitioner.

This is an RCT where data on adverse drug reactions and events including stroke and readmission will be collected. It is possible that differences can be determined between the two groups before the recruitment 
Table 2 Schedule of enrolment, interventions and assessments for patient-centred educational exchange (PCEE) to improve patients' self-management of medications after a stroke

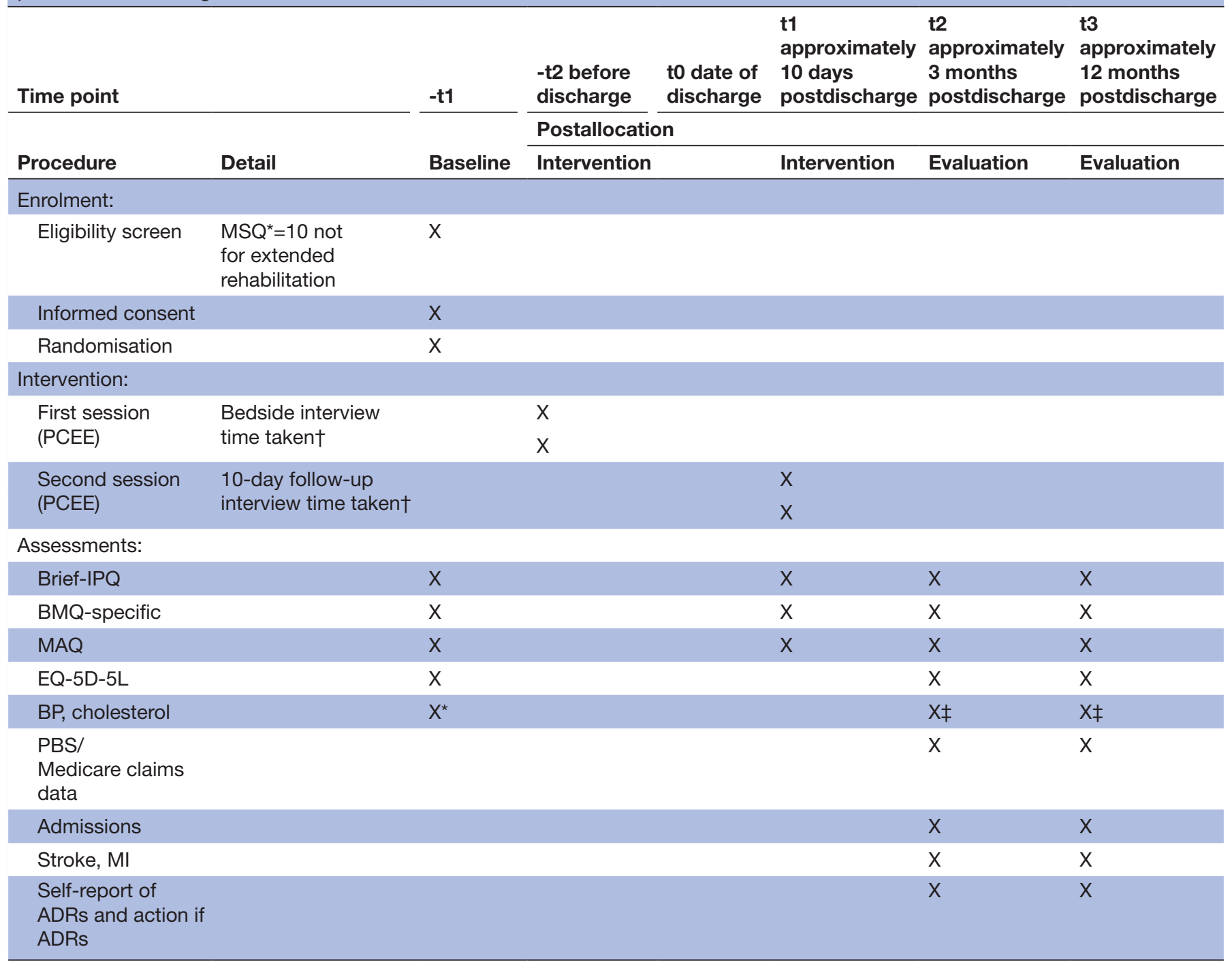

${ }^{*}$ These are standard care clinical tests performed as part of routine patient care.

†Time taken for PCEE.

†Self reported by participants.

ADR, adverse drug event; BMQ specific, Beliefs about medicines Questionnaire ${ }^{25}$; BP, blood pressure; Brief IPQ, Brief IIIness Perception Questionnaire ${ }^{24}$; EQ-5D-5L, Quality of Life Measure ${ }^{42}$; MAQ, Medication Adherence Questionnaire ${ }^{26}$; MI, myocardial infarction; MSQ, Mental State Questionnaire ${ }^{33}$; PBS/Medicare claims data, Dispensing data obtained from the Australians Pharmaceutical Benefits Scheme.

period is complete. A data safety monitoring committee (consisting of an independent medical doctor-clinical pharmacologist and pharmacist-Drug Use Evaluation Pharmacist) has been established to analyse the adverse events every 6 months with responsibility to terminate recruitment into the study early if necessary.

This RCT will provide evidence about the effect of a PCEE on patient adherence, self-reported medication-taking behaviour, clinical outcomes, quality of life, changes in knowledge and beliefs towards medicines and illness. It is expected that communication of results will inform an evidence-based approach to communication with patients about medication-taking behaviour related to stroke prevention. Communication of results of this study will seek to impact on the practice of health practitioners and consumers interested in patient medicine-taking behaviour and those interested in secondary prevention of stroke.

\section{ETHICS AND DISSEMINATION}

Participants will be provided with information about the study and asked if they consent to the study; 'Participant information and consent form' (see online supplementary file 3. Patient Information and Consent Form). To obtain consent for medication refill data from the PBS and occasions of service by visits to doctor data from Medicare, the patients will be given an extra consent 
form as required by the Department of Human Services. This is also contained in the 'Participant information and consent form'. The patient can choose not to supply the extra consent for access to PBS/Medicare data. The participant is free to withdraw from the study at any time.

Acknowledgements The authors would like to acknowledge the feedback and advice provided by participants of the feasibility study conducted to inform this protocol. The authors would like to acknowledge the encouragement and feedback from the Medical and Nursing Staff of the Stroke Unit at Princess Alexandra Hospital during the development of this protocol. The authors would like to acknowledge Shannon Doyle for the artwork used in the infographics.

Contributors JAC, WNC, DR and JAW were all equally involved in the development of this protocol. DR provided advice on academic detailing. JAW provided advice on Quality of Life measurement and economic analysis. JAC and WNC wrote the initial drafts of this paper. All authors involved in the reviewing and editing, and approval of the final protocol manuscript.

Funding The Research Strategies Funding, School of Pharmacy, University of Queensland has provided funding for the first 3-month Pharmaceutical Benefits Scheme/Medicare claims data for the first 60 participants. Further, funding is being sought to pay for the remaining claims data.

Competing interests None declared.

Patient consent Not required.

Ethics approval The trial has ethics approval from Metro South Human Research Ethics Committee (HREC/15/QPAH/531) and The University of Queensland Institutional Human Research Ethics (2015001612).

Provenance and peer review Not commissioned; externally peer reviewed.

Open access This is an open access article distributed in accordance with the Creative Commons Attribution Non Commercial (CC BY-NC 4.0) license, which permits others to distribute, remix, adapt, build upon this work non-commercially, and license their derivative works on different terms, provided the original work is properly cited, appropriate credit is given, any changes made indicated, and the use is non-commercial. See: http://creativecommons.org/licenses/by-nc/4.0/.

\section{REFERENCES}

1. Australian Bureau of Statistics. Australia's leading causes of death, 2016. 2018 http://www.abs.gov.au/ausstats/abs@.nsf/Lookup/ by\%20Subject/3303.0 2016 Main\%20Features Australia's\%20 leading\%20causes\%20of\%20death,\%202016 3 (accessed 8 Jul 2018).

2. Hankey GJ. The global and regional burden of stroke. Lancet Glob Health 2013;1:e239-e240.

3. Feigin VL, Forouzanfar MH, Krishnamurthi R, et al. Global and regional burden of stroke during 1990-2010: findings from the Global Burden of Disease Study 2010. Lancet 2014;383:245-55.

4. Australian Institute of Health and Welfare. Australia's Health 2016. Australia's health series. Canberra: AlHW, 2016.

5. Hardie K, Hankey GJ, Jamrozik K, et al. Ten-year risk of first recurrent stroke and disability after first-ever stroke in the Perth Community Stroke Study. Stroke 2004;35:731-5.

6. Burn J, Dennis M, Bamford J, et al. Long-term risk of recurrent stroke after a first-ever stroke. The Oxfordshire Community Stroke Project. Stroke 1994:25:333-7.

7. Hankey GJ. Long-term outcome after ischaemic stroke/transient ischaemic attack. Cerebrovasc Dis 2003;16 Suppl 1(Suppl. 1):14-19.

8. Goldstein LB, Bertels C, Davis JN. Interrater reliability of the NIH stroke scale. Arch Neurol 1989;46:660-2.

9. Coull AJ, Lovett JK, Rothwell PM, et al. Population based study of early risk of stroke after transient ischaemic attack or minor stroke: implications for public education and organisation of services. BMJ 2004;328:326-8.

10. Powers WJ, Rabinstein AA, Ackerson T, et al. 2018 Guidelines for the Early Management of Patients With Acute Ischemic Stroke: A Guideline for Healthcare Professionals From the American Heart Association/American Stroke Association. Stroke 2018;49:e46-e99.

11. Intercollegiate Stroke Working Party. National Clinical Guideline for Stroke. 5th Edition. London: edRoyal College of Physicians, 2016.

12. Scottish Intercollegiate Guidelines Network. Management of patients with stroke or TIA:assessment, investigation, immediate management and secondary prevention. Scottish Intercollegiate
Guidelines Network Hillside Crescent. Edinburgh: Scottish Intercollegiate Guidelines Network, Elliott House, 2008:8-10.

13. Stroke Foundation. Clinical Guidelines for Stroke Management 2017. Melbourne Australia.: Stroke Foundation, 2017.

14. Kronish IM, Diefenbach MA, Edmondson DE, et al. Key barriers to medication adherence in survivors of strokes and transient ischemic attacks. J Gen Intern Med 2013;28:675-82.

15. Bushnell CD, Olson DM, Zhao X, et al. Secondary preventive medication persistence and adherence 1 year after stroke. Neurology 2011;77:1182-90.

16. Glader EL, Sjölander M, Eriksson M, et al. Persistent use of secondary preventive drugs declines rapidly during the first 2 years after stroke. Stroke 2010;41:397-401.

17. Runciman WB, Roughead EE, Semple SJ, et al. Adverse drug events and medication errors in Australia. Int J Qual Health Care 2003;15 Suppl 1(Supplement 1):49i-59.

18. Jiang $Y$, Yang $X$, Li Z, et al. Persistence of secondary prevention medication and related factors for acute ischemic stroke and transient ischemic attack in China. Neurol Res 2017;39:492-7.

19. Jamison J, Sutton S, Mant J, et al. Barriers and facilitators to adherence to secondary stroke prevention medications after stroke: analysis of survivors and caregivers views from an online stroke forum. BMJ Open 2017;7:e016814.

20. Ellis G, Rodger J, McAlpine C, et al. The impact of stroke nurse specialist input on risk factor modification: a randomised controlled trial. Age Ageing 2005;34:389-92.

21. McManus JA, Craig A, McAlpine $C$, et al. Does behaviour modification affect post-stroke risk factor control? Three-year followup of a randomized controlled trial. Clin Rehabil 2009;23:99-105.

22. Adie K, James MA. Does telephone follow-up improve blood pressure after minor stroke or TIA? Age Ageing 2010;39:598-603.

23. O'Carroll RE, Chambers JA, Dennis M, et al. Improving adherence to medication in stroke survivors: a pilot randomised controlled trial. Ann Behav Med 2013;46:358-68.

24. Broadbent E, Petrie KJ, Main J, et al. The brief illness perception questionnaire. J Psychosom Res 2006;60:631-7.

25. Horne R, Weinman J, Hankins M. The beliefs about medicines questionnaire: The development and evaluation of a new method for assessing the cognitive representation of medication. Psychol Health 1999;14:1-24.

26. Morisky DE, Green LW, Levine DM. Concurrent and predictive validity of a self-reported measure of medication adherence. Med Care 1986;24:67-74.

27. Nguyen TM, La Caze A, Cottrell N. Validated adherence scales used in a measurement-guided medication management approach to target and tailor a medication adherence intervention: a randomised controlled trial. BMJ Open 2016;6:e013375.

28. Soumerai SB, Avorn J. Principles of educational outreach ('academic detailing') to improve clinical decision making. JAMA 1990;263:549-56.

29. O'Brien MA, Rogers S, Jamtvedt G, et al. Educational outreach visits: effects on professional practice and health care outcomes. Cochrane Database Syst Rev 2007;308 http://onlinelibrary.wiley.com/doi/ http:// onlinelibrary.wiley.com/store/10.1002/14651858.CD000409.pub2/ asset/CD000409.pdf? $v=1 \& t=h h 9 \times h 6 b h \& s=09 d b 68 c 95091 \mathrm{bda} 34 \mathrm{e} 3 \mathrm{~b}$ 4e28ad775312ec107ff5.

30. West CM, Dodd MJ, Paul SM, et al. The PRO-SELF(c): Pain Control Program--an effective approach for cancer pain management. Oncol Nurs Forum 2003;30:65-73.

31. Abernethy AP, Currow DC, Shelby-James T, et al. Delivery strategies to optimize resource utilization and performance status for patients with advanced life-limiting illness: results from the "palliative care trial" [ISRCTN 81117481]. J Pain Symptom Manage 2013;45:488-505.

32. Coombes J, Rowett D, Whitty J, et al. A Conversation About Stroke Medications: Using Patient Perceptions to Personalise Educational Messages. Cerebrovasc Dis 2016;42(Suppl 1):100.

33. Pfeiffer E. A short portable mental status questionnaire for the assessment of organic brain deficit in elderly patients. J Am Geriatr Soc 1975;23:433-41.

34. SealedenvelopeLTD. sealed envelope London: Clerkenwell Workshops 2015, 2015. (accessed 17 Jul 2015).

35. Nguyen VHV, Poon J, Tokuda L, et al. Pharmacist telephone interventions improve adherence to stroke preventive medications and reduce stroke risk factors: A randomized controlled trial. Stroke 2011;42:e244.

36. Ho PM, Lambert-Kerzner A, Carey EP, et al. Multifaceted intervention to improve medication adherence and secondary prevention measures after acute coronary syndrome hospital discharge: a randomized clinical trial. JAMA Intern Med 2014:174:186-93. 
37. Bailey JE, Wan JY, Tang J, et al. Antihypertensive medication adherence, ambulatory visits, and risk of stroke and death. J Gen Intern Med 2010;25:495-503.

38. Hedegaard U, Kjeldsen LJ, Pottegård A, et al. Multifaceted intervention including motivational interviewing to support medication adherence after stroke/transient ischemic attack: a randomized trial. Cerebrovasc Dis Extra 2014;4:221-34.

39. Lehmann EL, D'Abrera HJM. Nonparametrics: statistical methods based on ranks / E. L. Lehmann, with the special assistance of H. J. M. D'Abrera. San Fransisco, 1998:76-81.

40. Stroke Foundation. My Stroke Journey Melbourne: Stroke Foundation. 2017;18 https://strokefoundation.org.au/about-stroke/ help-after-stroke/stroke-resources-and-fact-sheets2018.

41. Lum E, Muscillo N, McLeod S, et al. Medication Reconciliation-the Queensland Health Experience. Journal of Pharmacy Practice and Research 2007;37:7-10.

42. Euroqol Research Foundation. EQ-5D-5L User Guide. 2015 https:// euroqol.org/wp-content/uploads/2016/09/EQ-5D-5L_UserGuide_ 2015.pdf (accessed 27/1/18 2018).

43. Herdman M, Gudex C, Lloyd A, et al. Development and preliminary testing of the new five-level version of EQ-5D (EQ-5D-5L). Qual Life Res 2011;20:1727-36.

44. Norman R, Cronin P, Viney R. A pilot discrete choice experiment to explore preferences for EQ-5D-5L health states. Appl Health Econ Health Policy 2013;11:287-98.
45. Shay LA, Lafata JE. Where is the evidence? A systematic review of shared decision making and patient outcomes. Med Decis Making 2015;35.

46. O'Carroll RE, Chambers JA, Dennis M, et al. Improving medication adherence in stroke survivors: mediators and moderators of treatment effects. Health Psychol 2014;33:1241-50.

47. Lehmann A, Aslani P, Ahmed R, et al. Assessing medication adherence: options to consider. Int J Clin Pharm 2014;36:55-69.

48. Steiner JF, Prochazka AV. The assessment of refill compliance using pharmacy records: methods, validity, and applications. J Clin Epidemiol 1997;50:105-16.

49. Bijlsma MJ, Janssen F, Hak E. Estimating time-varying drug adherence using electronic records: extending the proportion of days covered (PDC) method. Pharmacoepidemiol Drug Saf 2016;25:325-32.

50. Bryson CL, Au DH, Young B, et al. A refill adherence algorithm for multiple short intervals to estimate refill compliance (ReComp). Med Care 2007; 45:497-504.

51. Nguyen TM, La Caze A, Cottrell N. What are validated self-report adherence scales really measuring?: a systematic review. $\mathrm{Br} \mathrm{J}$ Clin Pharmacol 2014;77:427-45.

52. Rozich JD, Haraden CR, Resar RK. Adverse drug event trigger tool: a practical methodology for measuring medication related harm. Qual Saf Health Care 2003;12:194-200.

53. Golicki D, Niewada M, Karlińska A, et al. Comparing responsiveness of the EQ-5D-5L, EQ-5D-3L and EQ VAS in stroke patients. Qual Life Res 2015;24:1555-63. 